Journal of Research in Interprofessional

Practice and

Education

Vol. 4.2

September 2014

\section{Action, Reflection, and Evolution: A Pilot Implementation of Interprofessional Education across Three Rehabilitation Disciplines}

\author{
Teresa Paslawski, PhD; Renate Kahlke, MA; Tara Hatch, BSW, RSW; \\ Mark Hall, PhD; Lu-Anne McFarlane, MSc, R-SLP; Barbara Norton, \\ BScPT; Elizabeth Taylor, PhD; Sharla King, PhD
}

\begin{abstract}
Background: Interprofessional collaboration (IPC) is accepted as standard practice in healthcare. Because of this expectation, there is an increased need for growth in interprofessional education (IPE). Despite this need, the scholarship of IPE is limited. To better understand the challenges of IPE and improve on future endeavours, this article describes an IPE collaboration that was less successful, and the conclusions drawn from team reflection regarding IPE. We report on the challenges and the lessons learned.

Methods and Findings: After one year of an IPE pilot project, the research team conducted a reflection exercise involving three iterations: 1) initial group meeting to discuss reflection questions, 2) individual review of meeting notes, 3) subsequent group meeting to confirm accuracy of the data. The confirmed data were then analyzed using thematic analysis.

Conclusions: The key themes that emerged regarding the limited success of the pilot were focused on communication-between members of the research team, with the students, and with other faculty impacted by the pilot. Recommendations regarding improvements to facilitate future IPE initiatives are discussed. The summary conclusion of our exercise acknowledged that as IP educators we must remain vigilant to demonstrate IPC in the same manner as we teach it.
\end{abstract}

Keywords: Interprofessional education (IPE); Interprofessional collaboration (IPC); Reflection

\section{Background}

Interprofessional education (IPE), "when two or more professions learn about, from, and with each other to enable effective collaboration and improve health outcomes," is recognized as an integral component in preparing professionals to work effectively in collaborative healthcare environments [1]. Yet, despite its importance, an examination of the development of IPE reveals challenges that have hampered its growth [2], reflecting an observation made by the World Health Organization that "interprofessional education and collaborative practice can be difficult concepts to explain, understand and implement" [1]. Fourgner and Habib [3] admit that the development and delivery of an IPE curriculum is complex and may be more difficult than single-discipline courses or programs. In keeping with this observation, Hammick and colleagues [4] identify authenticity and customization of IPE as requisite in the creation of an IPE curriculum to ensure success from the perspective of both learners and teachers or facilitators from each of the disciplines involved. 
2

IPE Pilot: Action, Reflection, and Evolution

Paslawski, Kahlke, Hatch, Hall, McFarlane, Norton, Taylor, \& King
Journal of Research in Interprofessional Practice and Education

Vol. 4.2

September 2014
The IPE literature is saturated with descriptions of educational experiences or curricular innovations that have brought pre-licensure students together in teams to learn "from, with and about each other" [5]. The published scenarios are fairly standard; faculty members from different disciplines came together, developed an interprofessional (IP) educational experience, determined that students believed it to be a positive experience, and concluded that more IPE needs to occur. Generally, all published efforts to develop and integrate IPE are presented as successful. In contrast, this article describes a collaboration that was less than successful, as measured by traditional academic means. What follows is a discussion of a pilot IPE project cut short, and the lessons learned that will guide preparation for the next stage in promoting IPE across our campus. It focuses on documenting experiences, rather than developing experiences, and reports on the challenges and the lessons learned. Reeves and Freeth [6] observed that there is value in reflection and planning for the purpose of improving IP initiatives. Because of the challenges experienced in this collaboration, the desire arose to share our experience of evaluating and documenting IP competencies in an academic setting.

\section{Action: The pilot study}

As a step toward developing and implementing IPE across disciplines within a large, research-intensive post-secondary institution, a pilot study was conducted in the Faculty of Rehabilitation Medicine to examine the utility of the Interprofessional Learning Pathway ("the Pathway"). The Pathway was developed from a strong theoretical and process-oriented foundation using participatory action research. However, developing tools to operationalize the Pathway highlighted challenges not initially anticipated by the IP team involved in this study. This article examines the barriers to successful implementation of this project as a model for preparation in other IP endeavours.

\section{The interprofessional learning pathway}

The Interprofessional Learning Pathway is an initiative developed by the Health Sciences Education and Research Commons, which seeks to recognize and track the collection of IP learning experiences threaded throughout each health science program at our institution. The objectives of the Pathway are to provide guidance regarding IPE and to recognize students' achievement of IP competencies through classroom-based, clinical, and co-curricular experiences. This approach seeks to capitalize on learning that already occurs but which may not be identified as IP learning, as opposed to "adding on" modules or IPE experiences, as would typically occur. The Pathway is operationalized in a competency framework that outlines four core competencies identified as critical to IP practice: communication, collaboration, role clarification, and reflection, with patient-centred care woven throughout. This set of competencies provides a practical means of integrating the IP competencies into curricula, and it facilitates individual tracking of IP experiences and achievement of IP competencies. Thus, the Pathway seeks to engage students and faculty members in reflecting on their IP experiences as they occur, thereby 
3

IPE Pilot: Action, Reflection, and Evolution

Paslawski, Kahlke, Hatch, Hall, McFarlane, Norton, Taylor, \& King

Journal of Research in Interprofessional Practice and Education

Vol. 4.2

September 2014 solidifying those experiences as IP education. A detailed description of the Interprofessional Learning Pathway is available online [7].

\section{Pilot Study: Documenting Use of the IP Learning Pathway}

The pilot study involved using the Pathway to identify existing IPE experiences within the programs of three health disciplines in the Faculty of Rehabilitation Medicine-occupational therapy, physical therapy, and speech language pathology-from the perspective of students and faculty. The Faculty of Rehabilitation Medicine was identified as the pilot faculty for two reasons. First, all three of the programs are delivered in a condensed, two-year master's degree format, offering multiple opportunities for evaluation. Second, because advocates of IP are considered important to IPE education success [8], a core team of IP champions was identified in the Faculty to facilitate its implementation.

In addition to documenting the development of IP competencies via the IP Learning Pathway, the pilot was also expected to help the researchers refine a tool to track acquisition of IP skills and to evaluate them in courses and clinical practica. The intended deliverables were a database that would highlight where IP educational opportunities were occurring, and a validated tool through which students could assess their knowledge and skills in each of the four IP competencies: role clarification, communication, collaboration, and reflection. These competencies were identified from a critical review of the IP literature as well as through discussion with professional associations, training centres, and the Canadian Interprofessional Health Collaborative [9-13]. The competencies for the Pathway are defined as follows:

- role clarification - understanding of one's own role and the roles of others in an IP context

- communication - IP team process skills that achieve common goals

- collaboration - IP team process skills that achieve common goals

- reflection - critical evaluation of professional and team practice in an IP context to enhance patient care

The proposed pilot was planned to be conducted over two years and included input from students and faculty through online surveys, student completion of a self-assessment learning record, focus groups of students, and interviews or focus groups of faculty. The pilot team included faculty members from each of the three health disciplines and members of the Health Sciences Education and Research Commons. Approval for the study was obtained from the University's ethics board prior to initiation, and informed consent was obtained from students prior to data collection. Students who agreed to participate in the study were required to complete three components: 1) answer four questions, early in their program and again at the end of their first year, related to their understanding of a patient-centred care team and the role of their profession as a member of a care team, 2) submit an Interprofessional Learning Record, a template designed to track IP education and a student's progression in competency achievement, at the end of each term, and 3) 
4

IPE Pilot: Action, Reflection, and Evolution

Paslawski, Kahlke, Hatch, Hall, McFarlane, Norton, Taylor, \& King
Journal of Research in Interprofessional Practice and Education

Vol. 4.2

September 2014 participate in focus group interviews at the end of the school year. Faculty members who teach in each of the three Rehabilitation Medicine departments were requested to participate in this study and asked to do the following: 1) at the end of each term, identify the interprofessional competencies they believe were taught in their course and document them using the interprofessional learning record, and 2) participate in focus groups to enable researchers to better understand how interprofessional competencies are taught in each curriculum.

The pilot project was terminated at the end of the first year on the consensus of the team. At the end of one year, limited data had been collected from students $(N=57)$. Only $10 \%$ of occupational therapy (OT) students $(N=13), 25 \%$ of physical therapy $(\mathrm{PT})$ students $(N=27)$ and $27 \%$ of speech language pathology (SLP) students $(N=17)$ completed both parts 1 and 2 , and no students participated in the focus group. No data had been collected from faculty-only one faculty member responded to email invitations to participate in the study. The decision to end the pilot was based on the paucity of data collected, project management concerns, and a desire by the research team to understand and improve processes before re-initiating the project.

\section{Methods and findings: Reflection and evolution-lessons learned}

To identify the strengths and weaknesses of the pilot, the team initiated a reflection exercise $[6,14]$ based on the principles of action research, a qualitative method that involves action, observation, and reflection with the goal of improving practice [15], conducted by those involved in the practice. The action was the implementation of the pilot project. The observations included both the data obtained from the pilot and the commentary of the members of the team involved in the pilot project. The members of the research team used a set of questions regarding the pilot process and outcome to guide their reflection. The questions were:

- What did we learn from the information that we gathered from the students?

- What did we learn from our approach to gathering information from faculty?

- What impacted our project outcomes?

The reflection involved several iterations. There was an initial group discussion of the questions - a brainstorming session during which responses were recorded. Each individual was then given the opportunity to read the combined notes from the session and add to or expand on it in a written format. These comments were submitted to the Health Sciences Education and Research Commons administrative co-ordinator to ensure a level of anonymity and that all team members' voices would be heard. A compilation of all of the comments was then provided to each team member. A follow-up meeting of the group confirmed that the responses captured the individual and collective reflections of the team members. Responses were then analyzed by two of the team members to identify themes that adequately represent the observations and reflections. These themes and representative quotes 
5

IPE Pilot: Action, Reflection, and Evolution

Paslawski, Kahlke, Hatch, Hall, McFarlane, Norton, Taylor, \& King
Journal of Research in Interprofessional Practice and Education

Vol. 4.2

September 2014 were shared with the eight core team members and refined until all team members were in agreement regarding the themes.

Consistent with the literature indicating that communication competencies are core to effective IPE [16-18], the key themes that emerged regarding the limited success of the pilot were focused on communication. The key themes were: 1) communication and co-ordination of the research team, 2) communication with students-understanding the lexicon of interprofessional collaboration, and 3) adapting to faculty needs-altering communication and allowing for flexibility of integration.

Team communication may require more explicit operationalizing than previous literature would have led us to believe. While it is recognized that communication is not mutually exclusive of "identification of roles and responsibilities" or of the larger issue of team development, the data suggest specific concerns of this collaborative team reinforce the value of focusing explicitly on communication, and they highlight specific issues of communication that are particularly pertinent to interprofessional collaboration. What follows is a discussion of each theme with accompanying quotes from our team reflection exercise presented in italics to illustrate each theme.

\section{Theme 1: Communication and co-ordination of the research team}

The first theme identified related to team process, specifically, communication and co-ordination. Despite the premature conclusion of the pilot, the most frequently cited positive aspect of the project that emerged was team attitude. It was agreed that members of the team were motivated to participate in the project and positively oriented to the process. This is consistent with the IP literature, which highlights the importance of champions of interprofessional collaboration in IP initiatives $[1,8]$.

Despite the enthusiasm and positive perspective of the team, members of the team emphasized that support in the form of a project manager and administrative support would have greatly facilitated the project.

"It was fun to come to meetings. The group is fun, positive, and open to ideas. Regardless of the outcome, I enjoyed the process."

"Don't assume that enthusiasm will take care of things or that good intentions will translate into action."

"Ideally this project shouldn't be an add-on. It needs to be flexible enough to fit into an existing framework or exercise within each department."

"Even when individuals committed to process, collaboration is a challenge and often an add-on."

This finding is aligned with reports of others who reiterate that IPE requires considerable time to develop and conduct $[6,19,20]$; the need for dedicated resources and project co-ordination has also been identified elsewhere in relation to IP implementation in clinical units [e.g., 21]. All too often IP activities are add-ons to exist- 
6

IPE Pilot: Action, Reflection, and Evolution

Paslawski, Kahlke, Hatch, Hall, McFarlane, Norton, Taylor, \& King
Journal of Research in Interprofessional Practice and Education

Vol. 4.2

September 2014 ing workloads. Whether this is in the academic or clinical environment, champions of IP practice take on these extra activities because they believe in the importance of collaboration, but often they don't have the time or resources to be able to achieve all of their objectives. In this project, the researchers involved worked well together as a cohesive group; however, process deficits were so significant that the relationship alone could not carry the load needed for optimal results. The group believed that the pilot suffered from issues of organizing project activities, setting and maintaining timelines and goals, and maintaining momentum, all of which would have been facilitated by appropriate resources or the efforts of a co-ordinator for whom this was part of the job and not an add-on. The pilot had no dedicated resources and took place in addition to the research team members' regular workload. In the end, this proved too much to absorb by the team and was one of the factors that led to the termination of the study at the one-year mark.

It was also agreed that there was a lack of clarity regarding roles, expectations, and responsibilities of each of the team members.

"We need to clearly identify roles for each person ... in order to ensure that things get done. I think things have gone uncompleted because nobody was really 'the person' responsible for them; also, sometimes all the tasks fell on one person."

"We need practical support-someone to do the work-OR we need to be explicit in terms of action items, what each person will do."

"Variations in participation [in the development and execution of the pilot] may shape perceptions of process."

An assumption may have been made that since we all wanted to participate, the project would succeed; therefore, a discussion on foundational team process components was assumed to be unnecessary. This erroneous assumption speaks to the need to attend to components of team development, such as clear communication, role clarification, and team expectations. A firm foundation in the basic competencies of interprofessional collaboration (IPC) [22] is important for all participants, champions included.

In addition, responses noted a lack of clarity with respect to the project goals. "I was sometimes confused about what kind of data we were trying to collect and why. ... I wasn't always clear what was being collected and how our methods aligned with what we were trying to find out." Given that the approach taken in the pilot was a novel one, it was difficult to determine exactly where the project could and should lead. Testing the tools and approaches in advance of the project may have clarified which goals could be accomplished within the scope of the project.

Theme 2: Communication with the students-understanding the lexicon of interprofessional collaboration

The second theme that emerged was the communication needs of students. Students require practical language and explicitly discussed common terminology 
7

IPE Pilot: Action, Reflection, and Evolution

Paslawski, Kahlke, Hatch, Hall, McFarlane, Norton, Taylor, \& King

Journal of Research in Interprofessional Practice and Education

Vol. 4.2

September 2014 in order to self-identify and document IP competencies. "Should be practical, concrete examples" and "connections [made to] IP competencies." The pilot team agreed that clear, practical language would have assisted with explaining the tool and how students could use the IP Pathway tools to track their interprofessional learning experiences. We wondered if "collaborationese," the academic language of collaboration, interfered with ease of use of the tool. Specifically, we asked ourselves the following questions: Is the culture of collaboration, presented from the perspective of IP enthusiasts, exclusionary? Do we all interpret the language of collaboration in the same way in each of our disciplines? Is there a need to explicitly teach the language of collaboration, in the same way that other components of collaboration such as team dynamics must be explicitly addressed? Or, should we, as champions of collaboration, endeavour to avoid collaborationese? "Consider using a form that has real-life examples that they can fill out, and then we translate it into 'collaborationese." Terms that could be identified as collaborationese, which may have multiple or obscure meaning for novice collaborators, include, for example, shared decision-making, team outcomes, conflict management models, active listening, reflection, and even collaboration.

Student responses to the pathway tools indicated variable understanding of the competencies and the language of interprofessional teamwork. Some of this variability existed between students, though the research team also observed some differences in how students interpreted the IP competencies between programs. The interprogram difference may be attributed to differences in how the project was presented to students (e.g., differences were noted in student response based on the number of IPC champions and the level of their involvement when the project was presented to the students), differences in how IPE had been taught in their program (OT and PT students participate in a course on IPC whereas SLP students do not), and the language used in each discipline. Regardless, we were not successful in establishing a common language of collaboration, and the use of collaborationese appears to have negatively affected data collection in the pilot: "Competencies need to be integrated into discipline-specific documents, not an add-on.” It was agreed by the pilot team that future applications of the Pathway should include explicit discussion of the terminology of collaboration, simple and easy-to-use language, and consideration of the possible need for translation from discipline-specific to interprofessional language.

Additionally, the pilot tools used by students to document their IP competencies provided a challenge in two ways. First, the tools were formed around a set of defined IP competencies. This was done in an attempt to create a common language. However, as previously mentioned, the lack of discussion around the terminology and use of formal competency language resulted in wide variability in responses. Second, the process of gathering the data assumed that students would self-assess in order to reflect on their IP learning. Again, this did not seem to occur.

"If self-assessment is important in assessing student competence (and it may not be the only way), then we will need to anchor that 
8

IPE Pilot: Action, Reflection, and Evolution

Paslawski, Kahlke, Hatch, Hall, McFarlane, Norton, Taylor, \& King
Journal of Research in Interprofessional Practice and Education

Vol. 4.2

September 2014 self-assessment in concrete experiences, like a team meeting they experienced, a specific interaction with another professional, etc."

"They seem to have limited awareness of the IP exposure they are receiving in their courses OR had trouble linking that experience to the form that we provided."

It was agreed by the pilot team that a lack of understanding of IP language and a lack of student experience in self-assessment contributed to wide variability in students' self-assessments using the current pilot tool.

The literature supports the need to develop a common language, particularly in relation to IPE, as the discussion of language in the IP literature is primarily in relation to team development, not IPE. Atkinson and colleagues [23] identified the development of a common language as an important factor in interprofessional collaborations. Much of the discussion around a common language assumes early discussion and consensus building with respect to the language [24,25]. Our experience in this pilot and evidence from the literature supports the need to have an open discussion around the language of IP competencies and IPE.

Theme 3: Adapting to faculty needs-communication and flexibility of integration

The third theme related to communication with faculty was a need for flexibility when integrating the IP competencies into curricula. Different professions have different work practices and approaches to communication [20]. The same is true for the educational programs of the professions. Although our three departments operate in the same faculty, there are significant differences in the way programs are run and in the manner in which communication occurs. The original approach to implementing the Interprofessional Learning Pathway planned for flexibility in terms of how the framework tool would be used, assuming that this would facilitate use of the tool. However, the level of flexibility required for use of the tool was not conducive to a pilot study in terms of data collection. We endeavoured to conduct data collection at the same time and in the same manner across the three departments to obtain consistency in terms of the length of time that students were in training. In retrospect, this approach was not ideal given the differences across the programs with respect to time-tabling of courses and practical experiences. It is suspected that a longer time frame (e.g., the original two-year plan) but with greater flexibility regarding when and how data were collected would have yielded improved response rates and richer data.

In addition, the team indicated that it would have been beneficial to complete more groundwork in advance of the pilot. Although we met with department chairs at the outset, meetings with both instructors and senior administration in advance of the project may have illuminated the communication needs for each program, as well as suggestions for integrating Pathway tools in each program's curriculum: "Need to make it easy and not be asking more of them than necessary." It is also rec- 
9

IPE Pilot: Action, Reflection, and Evolution

Paslawski, Kahlke, Hatch, Hall, McFarlane, Norton, Taylor, \& King
Journal of Research in Interprofessional Practice and Education

Vol. 4.2

September 2014 ognized that time is required for meaningful change to take place in an academic setting, again highlighting the limitation of a one-year, one-time pilot.

\section{Conclusion}

In place of flexibility or openness, as interpreted by the team, regarding use of the Interprofessional Learning Pathway, a more effective approach may have been an initial dialogue with student participants. This dialogue could have included an explicit introduction to the language of collaboration as operationalized by the Pathway, followed by concrete examples of IPC recognized by the participants. Then flexibility would have been not at the front end, as initially considered, but at the back end, resulting in a richer understanding of how IPC is identified by students and faculty. Consequently, this approach would be anticipated to result in a richer description of the interprofessional language and expansion of the Pathway, to aid in broader application across health sciences disciplines.

The early termination of our pilot and the opportunity to purposefully reflect has highlighted a number of process- and tool-specific barriers that contributed to the outcome of the pilot and inform current and future IPE initiatives on campus. Communication was recognized as a central issue in process, including delineating roles and responsibilities of the members of the team and setting goals for components of the pilot. Ironically, collaborationese, the language of collaboration, appeared to impede understanding of the pathway and IP competencies and negatively affected the outcome.

It was acknowledged that IPC is resource-intensive and that a lack of resources, and a lack of time dedicated exclusively to IP work instead of IP activities always being an add-on, contributed significantly to the unsatisfactory conclusion of this pilot. But it is also important to acknowledge that this intensive and process-sensitive training requires vigilance on the part of those who promote it. Through the practice of reflection, one of the Pathway competencies, the other competencies we highlight for our students-role clarification, communication, and collaborationwere all identified as areas requiring continuing attention in order to be successful in IPE. We must walk the talk-a lesson learned.

\section{References}

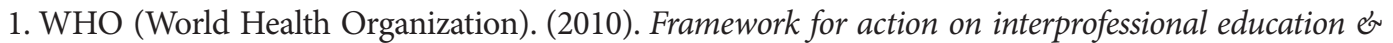

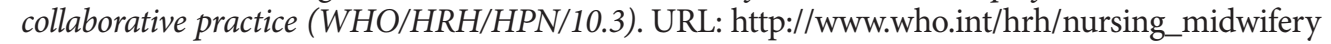
'Len', [November 03, 2010].

2. Clark, P.G. (2011). The devil is in the details: The seven deadly sins of organizing and continuing interprofessional education in the US. Journal of Interprofessional Care, 25(5), 321-327.

3. Fougner, M., \& Habib, L. (2008). If I had a rich picture ...: Insights into the use of "soft" methodological tools to support the development of interprofessional education. Journal of Interprofessional Care, 22(5), 488-498.

4. Hammick, M., Freeth, D., Koppel, I., Reeves, S., \& Barr, H. (2007). A best evidence systematic review of interprofessional education: BEME Guide no. 9. Medical Teacher, 29(8), 735-751.

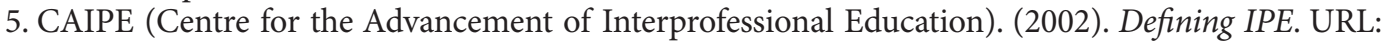
hthtp://wwww.caipe.org.uk/about-us/defining-ipe/!'November 03, 2010].

6. Reeves, S., \& Freeth, D. (2006). Re-examining the evaluation of interprofessional education for community mental health teams with a different lens: Understanding presage, process and product factors. Journal of Psychiatric and Mental Health Nursing, 13(6), 765-770. 
10

IPE Pilot: Action, Reflection, and Evolution

Paslawski, Kahlke, Hatch, Hall, McFarlane, Norton, Taylor, \& King
Journal of Research in Interprofessional Practice and Education

Vol. 4.2

September 2014

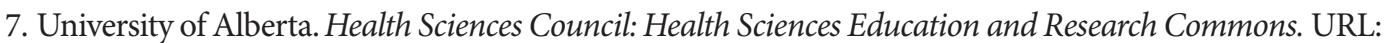

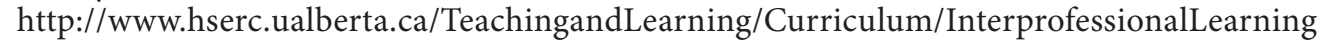
'Pathway.aspxi'i

8. Ho, K., Jarvis-Selinger, S., Borduas, F., Frank, B., Hall, P., Handfield-Jones, R., Hardwick, D.F., Lockyer, J., Sinclair, D., Lauscher, H.N., Ferdinands, L., MacLeod, A., Robitaille, M.A., \& Rouleau, M. (2008). Making interprofessional education work: The strategic roles of the academy. Academic Medicine, 83(10), 934-940.

9. Norman, G. R. (1985). Assessing clinical competence. New York: Springer.

10. Barr, H. (2005). Competent to collaborate: Towards a competency-based model for interprofessional education. Journal of Interprofessional Care, 12(2), 181-186.

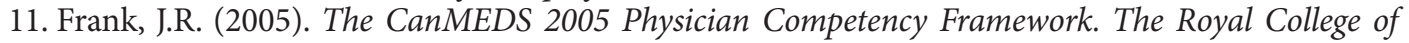

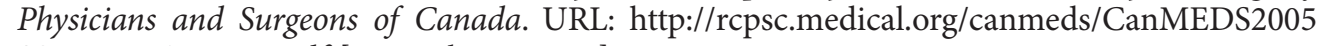
I/CanMEDS2005_e.pdfi'][December 7, 2010].

12. O'Neil, E.H., \& the Pew Health Professions Commission. (1998). Recreating health professional practice for a new century: The fourth report of the PEW Health Professions Commission. San Francisco: Pew Health Professions Commission.

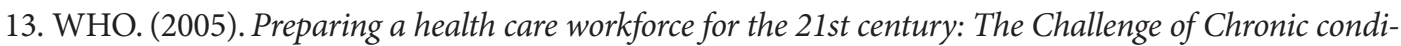
tions. URL:'http://www.who.int/chp/knowledge/publications/workforce_report/en/'][December $7,2010]$.

14. Garcia, L.M., \& Roblin, N.P. (2008). Innovation, research and professional development in higher education: Learning from our own experience. Teaching and Teacher Education, 24, 104-116.

15. Kemmis, S., \& McTaggart, R. (2000). Participatory action research. In Denzin, N., \& Lincoln, Y. (Eds.), Handbook of qualitative research, (pp. 567-605). London: Sage.

16. Mickan, S.M., \& Rodger, S.A. (2005). Effective health care teams: A model of six characteristics developed from shared perceptions. Journal of Interprofessional Care, 19(4), 358-370.

17. Vyt, A. (2008). Interprofessional and transdisciplinary teamwork in health care. Diabetes/Metabolism Research and Reviews, 24(S1), S106-S109.

18. Suter, E., Arndt, J., Arthur, N., Barboosingh, J., Taylor, E., \& Deutschlander, S. (2009). Role understanding and effective communication as core competencies for collaborative practice. Journal of Interprofessional Care, 23(1), 41-51.

19. Cooper, H., Carlisle, C., Gibbs, T., \& Watkins, C. (2001). Developing an evidence base for interdisciplinary learning: A systematic review. Journal of Advanced Nursing, 35(2), 228-237.

20. Kilminster, S., Hale, C., Lascelles, M., Morris, P., Roberts, T., Stark, P., Sowter, J., \& Thistlethwaite, J. (2004). Learning for real life: Patient-focused interprofessional workshops offer added value. Medical Education, 38(7), 717-726.

21. Vanderzalm, J., Hall, M.D., McFarlane, L., Rutherford, L., \& Patterson, S. (2013). Fostering interpro-

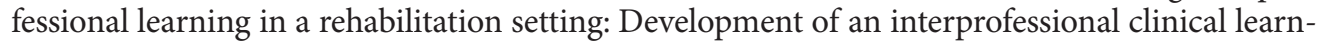

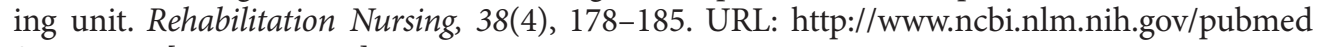
!23686422:[May 17, 2013].

22. Baerg, K., Lake, D., \& Paslawski T. (2012). Survey of interprofessional collaboration learning needs and training interest in health professionals, teachers, and students: An exploratory study. Journal of Research in Interprofessional Practice and Education, 2(2), 187-204.

23. Atkinson, M., Doherty, P., \& Kinder, K. (2005). Multi-agency working. Models, challenges and key factors for success. Journal of Early Childhood Research, 3(1), 7-17.

24. Salmon, G. (2004). Multi-agency collaboration: The challenges for CAMHS. Child and Adolescent Mental Health, 9(4), 156-161.

25. Lindeke, L.L., \& Siechert, A.M. (2005). Nurse-physician workplace collaboration. Online Journal of Issues in Nursing, 10(1), 92-102. 